PROCEEDINGS OF THE

AMERICAN MATHEMATICAL SOCIETY

Volume 130, Number 2, Pages 543-548

S 0002-9939(01)06102-0

Article electronically published on July 25, 2001

\title{
BOUNDED POINT EVALUATIONS FOR CYCLIC OPERATORS AND LOCAL SPECTRA
}

\author{
A. BOURHIM, C. E. CHIDUME, AND E. H. ZEROUALI
}

(Communicated by Joseph A. Ball)

\begin{abstract}
In this paper we study the concept of bounded point evaluations for cyclic operators. We give a negative answer to a question of L.R. Williams, Dynamic Systems and Applications 3 (1994), 103-112. Furthermore, we generalize some results of Williams and give a simple proof that nonnormal hyponormal weighted shifts have fat local spectra.
\end{abstract}

\section{INTRODUCTION}

Throughout this paper, $L(H)$ will denote the algebra of all linear bounded operators on a Hilbert space $H$. For an operator $S \in L(H)$, let $S^{*}$ denote its adjoint, $\sigma(S)$ its spectrum, $\sigma_{p}(S)$ its point spectrum, $\sigma_{a p}(S)$ its approximate spectrum, $\Gamma(S)$ its compression spectrum, $r(S)$ its spectral radius, $m(S)$ its lower bound (i.e., $\inf \{\|S x\|:\|x\|=1\})$ and $r_{1}(S)=\sup _{n} m\left(S^{n}\right)^{\frac{1}{n}}$ which equals $\lim _{n} m\left(S^{n}\right)^{\frac{1}{n}}$. For $F \subset \mathbb{C}$, we denote by $\bar{F}=\{\bar{z}: z \in F\}$ its conjugate set, $\operatorname{int}(F)$ its interior and $\operatorname{cl}(F)$ its closure.

Let $T \in L(H)$ be a cyclic operator on $H$ with cyclic vector $x$ that is the finite linear combinations of the vectors $x, T x, T^{2} x, \ldots$ are dense. A complex number $\lambda \in \mathbb{C}$ is said to be a bounded point evaluation of $T$ if there is a constant $M>0$ such that

$$
|p(\lambda)| \leq M\|p(T) x\|
$$

for every complex polynomial $p$. The set of all bounded point evaluations of $T$ will be denoted by $B(T)$. Note that it follows from the Riesz Representation Theorem that $\lambda \in B(T)$ if and only if there is a unique vector denoted $k(\lambda) \in H$ such that $p(\lambda)=\langle p(T) x, k(\lambda)\rangle$ for every complex polynomial $p$. By setting $q(z)=$ $(z-\lambda) p(z)$, we obtain $\lambda \in \overline{\sigma_{p}\left(T^{*}\right)}$. Conversely if $k(\lambda)$ is an eigenvector of $T^{*}$ associated to the eigenvalue $\bar{\lambda}$, we get $\langle p(T) x, k(\lambda)\rangle=p(\lambda)\langle x, k(\lambda)\rangle$. Hence $\langle x, k(\lambda)\rangle \neq 0$ and $\lambda \in \Gamma(T)$. Which gives

Proposition 1.1. $B(T)=\Gamma(T)=\overline{\sigma_{p}\left(T^{*}\right)}$.

An open subset $O$ of $\mathbb{C}$ is said to be an analytic set for $T$ if it is contained in $B(T)$ and if for every $y \in H$, the complex valued function $\hat{y}$, defined on $O$ by $\hat{y}(\lambda)=\langle y, k(\lambda)\rangle$ is analytic on $O$; equivalently, if $O \subset B(T)$ and if the function

Received by the editors July 10, 2000.

2000 Mathematics Subject Classification. Primary 47A10; Secondary 47B20.

(C)2001 American Mathematical Society 
$\lambda \longmapsto\|k(\lambda)\|$ is bounded on compact subsets of $O$ (see [10], Lemma 1.2). The largest analytic set for $T$ will be denoted by $B_{a}(T)$ and every point of it will be called analytic bounded point evaluation for $T$. Tavan Trent proved in $[9]$ that for cyclic subnormal operators, we have

$$
\Gamma(T) \backslash \sigma_{a p}(T)=B_{a}(T) .
$$

L.R. Williams proved in [10], Proposition 1.3, that for every arbitrary cyclic operator $T \in L(H), \Gamma(T) \backslash \sigma_{a p}(T) \subset B_{a}(T)$ and asked if $(*)$ remains valid for arbitrary cyclic operators ([10], Question A).

In section 2, we first give necessary and sufficient conditions for weighted shifts to satisfy $(*)$ and we exhibit an operator which provides a negative answer to the question of Williams. Then, in section 3, we devote our attention to operators with fat local spectra. Furthermore, we generalize Theorem 2.1 of [10] to the case of operators satisfying Dunford Condition $C$ and with no eigenvalues. Finally, we give a simple proof of Theorem 2.5 of [11] using the Zero-principle for analytic functions.

\section{Analytic Bounded point eValuations FOR UNILATERAL WEIGHTED SHIFT}

In [7, A.L. Shields represented a weighted shift operator as an ordinary shift operator (that is, as "multiplication by $z$ ") on a Hilbert space of formal power series (in the unilateral case) or formal Laurent series (in the bilateral case). He defined the concept of bounded point evaluations of a weighted shift to examine which power series and Laurent series represent analytic functions. In fact, this concept of bounded point evalutions for injective unilateral weighted shift coincides with the one defined above by Williams (see [10]).

We now describe the set of bounded point evaluations and the set of analytic bounded point evaluations for an arbitrary injective unilateral weighted shift. Let $S$ be a unilateral weighted shift on a Hilbert space $H$ with a positive weight sequence $\left(\omega_{n}\right)_{n \geq 0}$; that is,

$$
S e_{n}=\omega_{n} e_{n+1},
$$

where $\left(e_{n}\right)_{n \geq 0}$ is an orthonormal basis of $H$. Let $\beta$ be the following sequence given by

$$
\beta_{n}= \begin{cases}\omega_{0} \ldots \omega_{n-1} & \text { if } n>0 \\ 1 & \text { if } n=0\end{cases}
$$

The unilateral weighted shift $S$ is cyclic with cyclic vector $e_{0}$. It follows from Corollary 2 of [7] and Proposition 1.4 of [10] that the sets $B(S)$ and $B_{a}(S)$ have a circular symmetry about the origin. Also, it follows from Theorem 8 of [7] and Proposition 1.1 that $B(S)=\{0\}$ if $r_{2}(S)=0$; otherwise,

$$
\left\{\lambda \in \mathbb{C}:|\lambda|<r_{2}(S)\right\} \subset B(S) \subset\left\{\lambda \in \mathbb{C}:|\lambda| \leq r_{2}(S)\right\},
$$

where $r_{2}(S)=\liminf _{n \rightarrow+\infty}\left(\beta_{n}\right)^{\frac{1}{n}}$.

Theorem 2.1. If $r_{2}(S)>0$, then $B_{a}(S)=\left\{\lambda \in \mathbb{C}:|\lambda|<r_{2}(S)\right\}$. 
Proof. Let $\lambda \in B(S)$; then there is $k(\lambda) \in H$ such that $p(\lambda)=\left\langle p(S) e_{0}, k(\lambda)\right\rangle$ for every polynomial $p$. For every $n \geq 0$ we have

$$
\begin{aligned}
\left\langle e_{n}, k(\lambda)\right\rangle & =\left\langle\frac{1}{\beta_{n}} S^{n} e_{0}, k(\lambda)\right\rangle \\
& =\frac{\lambda^{n}}{\beta_{n}} .
\end{aligned}
$$

Hence, $k(\lambda)=\sum_{n \geq 0} \frac{\lambda^{n}}{\beta_{n}} e_{n}$ for every $\lambda \in B(S)$. Thus, the desired result holds.

For the weighted shift $S$, the spectrum $\sigma(S)$ is known to be the disk $\{\lambda \in$ $\mathbb{C}:|\lambda| \leq r(S)\}$, and the approximate point spectrum $\sigma_{a p}(S)$ is known to be the annulus $\left\{\lambda \in \mathbb{C}: r_{1}(S) \leq|\lambda| \leq r(S)\right\}$ where $r_{1}(S)=\lim _{n \rightarrow \infty}\left[\inf _{k \geq 0} \frac{\beta_{n+k}}{\beta_{k}}\right]^{\frac{1}{n}}$ (see [7]). Therefore,

$$
\Gamma(S) \backslash \sigma_{a p}(S)=\sigma(S) \backslash \sigma_{a p}(S)=\left\{\lambda \in \mathbb{C}:|\lambda|<r_{1}(S)\right\} .
$$

Hence we have the following theorem.

Theorem 2.2. Let $S$ be a unilateral weighted shift. Then, $\Gamma(S) \backslash \sigma_{a p}(S)=B_{a}(S)$ if and only if $r_{1}(S)=r_{2}(S)$.

A negative answer to Question A in [10] can be given by a unilateral weighted shift $S$ for which $r_{1}(S)<r_{2}(S)$. Let us consider an example of a such weighted shift. For $s \in \mathbb{N}$ there are unique $n, k \in \mathbb{N}$ such that $s=n !+k$ with $0 \leq k \leq(n+1) !-n !-1$. We set

$$
\beta_{s}=\beta_{n !+k}=e^{k} .
$$

Therefore, for every $k \in \mathbb{N}$, we have

$$
\frac{\beta_{k+1}}{\beta_{k}}= \begin{cases}e & \text { if } n ! \leq k<(n+1) !-1, \\ \frac{1}{e^{(n+1) !-n !-1}} & \text { if } k=(n+1) !-1 .\end{cases}
$$

Hence, the unilateral weighted shift $S$ corresponding to the weight $\left(\frac{\beta_{n+1}}{\beta_{n}}\right)_{n \geq 0}$ is bounded. For every $n \geq 2$, set $k_{n}=n !-n$. Clearly, we have $(n-1) ! \leq k_{n}<n$ ! and $\beta_{k_{n}}=e^{(n !-(n-1) !-n)}$. Therefore,

$$
\inf _{k \geq 0} \frac{\beta_{n+k}}{\beta_{k}} \leq \frac{\beta_{n+k_{n}}}{\beta_{k_{n}}}=\frac{1}{e^{(n !-(n-1) !-n)}} .
$$

Hence,

$$
r_{1}(S)=\lim _{n \rightarrow \infty}\left[\inf _{k \geq 0} \frac{\beta_{n+k}}{\beta_{k}}\right]^{\frac{1}{n}}=0 .
$$

On the other hand, it is clear that

$$
r_{2}(S)=\liminf _{n \rightarrow \infty}\left[\beta_{n}\right]^{\frac{1}{n}}=1 .
$$

Therefore, $B_{a}(S)=\{\lambda \in \mathbb{C}:|\lambda|<1\}$ and $\Gamma(S) \backslash \sigma_{a p}(S)=\emptyset$.

For the unilateral weighted shift $S$ the set of its analytic bounded point evaluations is exactly the interior of the set of its bounded point evaluations. This 
suggests the following question, which was posed by J.B. Conway in [2], page 65, for arbitrary cyclic subnormal operator.

Question. Does the interior of $B(T)$ always coincide with $B_{a}(T)$ for an arbitrary cyclic operator $T \in L(H)$ ?

\section{Local spectra of CYClic operators With Dunford's Condition C}

Let $T$ be a bounded operator on a complex Hilbert space $H$. For an element $x \in H$, let $\sigma_{T}(x)$ be its local spectrum and $\rho_{T}(x)$ be its local resolvent (see 11). For a closed subset $F$ of the complex plane $\mathbb{C}$, let $H_{T}(F)$ be the set of elements $x \in H$ such that $\sigma_{T}(x) \subset F$; it is a linear subspace of $H$. The operator $T$ is said to have the single valued extension property (svep) if zero is the unique vector $x \in H$ such that $\sigma_{T}(x)=\emptyset$, which is equivalent to that for every open set $U \subset \mathbb{C}$, the only analytic solution of the equation $(T-\lambda I) f(\lambda)=0$ for $\lambda \in U$ is the zero function $f \equiv 0$. The operator $T$ is said to satisfy Dunford's Condition C (DCC) if for every closed subset $F$ of $\mathbb{C}$, the linear subspace $H_{T}(F)$ is closed. It is known that every operator which satisfies DCC has the single valued extension property (see [1]). The operator $T$ is said to be hyponormal if $\left\|T^{*} x\right\| \leq\|T x\|$ for every $x \in H$; J.G. Stampfli [8] and M. Radjabalipour 4] have shown that hyponormal operators satisfy DCC. Recall that $T$ is said to be pure if the only reducing subspace $M$ of $T$ such that $T_{\mid M}$ is normal is $M=\{0\}$. In 10, Theorem 2.1, it is shown that for a pure cyclic hyponormal operator $T \in L(H)$ with cyclic vector $x, \sigma_{T}(S x)=\sigma(T)$ for every operator $S \in L(H)$ such that $S T=T S$ and $\operatorname{ker}\left(S^{*}\right)$ is finite dimensional. We generalize this result as follows.

Theorem 3.1. Let $T \in L(H)$ be a cyclic operator on $H$ with cyclic vector $x \in H$ and let $S \in L(H)$ be an operator on $H$ which commutes with $T$ such that $\operatorname{ker}\left(S^{*}\right)$ is finite dimensional. If $T$ satisfies DCC and $\sigma_{p}(T)=\emptyset$, then $\sigma_{T}(S x)=\sigma(T)$.

We prove this theorem using several lemmas. The following two lemmas are given in [10].

Lemma 3.2. Let $T \in L(H)$ be a cyclic operator on $H$ with a cyclic vector $x \in H$. If $T$ satisfies $D C C$, then $\sigma_{T}(x)=\sigma(T)$.

Lemma 3.3. Suppose that $H=H_{1} \oplus H_{2}$ where $H_{1}$ and $H_{2}$ are two Hilbert spaces such that $\mathrm{H}_{2}$ is finite dimensional. If $T$ has the single valued extension property and $H_{1}$ is an invariant subspace for $T$, then $A=T_{\mid H_{1}}$ has the single valued extension property and $\sigma_{A}(x)=\sigma_{T}(x)$ for every $x \in H_{1}$.

Lemma 3.4. Suppose that $H=H_{1} \oplus H_{2}$ where $H_{1}$ and $H_{2}$ are two Hilbert spaces such that $\mathrm{H}_{2}$ is finite dimensional. If $\mathrm{H}_{1}$ is an invariant subspace for an operator $T$ which satisfies $D C C$, then the operator $A=T_{\mid H_{1}}$ satisfies the DCC.

Proof. It follows from Lemma 3.3 that for every closed subset $F$ of $\mathbb{C}, H_{1_{A}}(F)=$ $H_{T}(F) \cap H_{1}$. So, the desired result holds.

Lemma 3.5. Suppose that $H=H_{1} \oplus H_{2}$ where $H_{1}$ and $H_{2}$ are two Hilbert spaces such that $H_{2}$ is finite dimensional. If $T \in L(H)$ is an operator without point spectrum such that $H_{1}$ is an invariant subspace for $T$, then $\sigma(T)=\sigma(A)$ where $A=T_{\mid H_{1}}$. 
Proof. We first write

$$
T=\left[\begin{array}{cc}
A & B \\
0 & C
\end{array}\right]
$$

with respect to the decomposition $H=H_{1} \oplus H_{2}$. Now, suppose that $A$ is invertible in $L\left(H_{1}\right)$, then $T H_{1}=H_{1}$. And so, $H_{1} \cap T H_{2}=\{0\}$ since $T$ is one-to-one. Let $x_{2} \in H_{2}$ such that $C x_{2}=0$; then $T x_{2}=B x_{2} \in H_{1} \cap T H_{2}$. Hence, $C$ is one-to-one and so, by the finite-dimensionality, $C$ is invertible. Therefore the operator $T$ is invertible with inverse given by

$$
\left[\begin{array}{cc}
A^{-1} & -A^{-1} B C^{-1} \\
0 & C^{-1}
\end{array}\right]
$$

Thus $\sigma(T) \subset \sigma(A)$. Conversely, it is known that $\sigma(A)=\bigcup_{x \in H_{1}} \sigma_{A}(x)$ (see [3], Theorem 1.9). It follows from Lemma 3.3 that $\sigma(A)=\bigcup_{x \in H_{1}} \sigma_{T}(x) \subset \sigma(T)$.

Proof of Theorem 3.1. Let $H_{1}$ be the closed linear subspace generated by $\left\{T^{n} S x\right.$ : $n \geq 0\}$. Then it is clear that $H_{1}$ is an invariant subspace for $T$. Since $T S=S T$ and $x$ is a cyclic vector for $T$, then $H_{1}=\operatorname{cl}(\operatorname{Im}(S))$. Therefore $H=H_{1} \oplus H_{2}$ where $H_{2}=\operatorname{ker}\left(S^{*}\right)$. It follows from Lemma 3.3 that $\sigma_{T}(S x)=\sigma_{A}(S x)$ where $A=T_{\mid H_{1}}$. Since $A$ is a cyclic operator with cyclic vector $S x$ and satisfies DCC (Lemma 3.4), it follows from Lemma 3.2 that $\sigma_{A}(S x)=\sigma(A)$. Since $\sigma_{p}(T)=\emptyset$, it follows from Lemma 3.5 that $\sigma(T)=\sigma(A)$. And so, $\sigma(T)=\sigma(A)=\sigma_{A}(S x)=\sigma_{T}(S x)$. The proof is complete.

Remark 3.6. Let $T \in L(H)$ be a cyclic operator with cyclic vector $x \in H$ and satisfies DCC such that $\sigma_{p}(T)=\emptyset$. For every nonzero polynomial $p, \sigma_{T}(p(T) x)=$ $\sigma(T)$. Therefore, $\sigma_{T}(y)=\sigma(T)$ holds for all $y$ in a dense subset of $H$. L.R. Williams proved in Theorem 2.5 of [1] that if $T$ is a nonnormal hyponormal (unilateral or bilateral) weighted shift operator, then $\sigma_{T}(x)=\sigma(T)$ for every nonzero element $x \in H$.

We give a simple proof of Theorem 2.5 of [11] using the fact that a nonzero analytic function has isolated zeros.

Theorem 3.7. Let $T$ be a nonnormal hyponormal weighted shift on $H$. Then for every nonzero element $x \in H, \sigma_{T}(x)=\sigma(T)$.

Proof. First suppose that $T$ is a nonnormal hyponormal unilateral weighted shift. Then $r(T)=r_{1}(T)=r_{2}(T)=\|T\|>0$ and by Theorem 2.1 we have $B_{a}(T)=$ $\operatorname{int}(\sigma(T))$. Now, let $x \in H$ such that there exists $\lambda \in \sigma(T) \backslash \sigma_{T}(x)$. So, there is vector valued analytic function $f$ on an open neighbourhood $V$ of $\lambda$ such that

$$
(T-\mu I) f(\mu)=x \quad \text { for every } \mu \in V .
$$

Since $\emptyset \neq V \cap \operatorname{int}(\sigma(T)) \subset B_{a}(T)$, then for every $\mu \in V \cap \operatorname{int}(\sigma(T))$ we have

$$
\begin{aligned}
\widehat{x}(\mu) & :=\langle x, k(\mu)\rangle \\
& =\langle(T-\mu I) f(\mu), k(\mu)\rangle \\
& =\left\langle f(\mu),(T-\mu I)^{*} k(\mu)\right\rangle \\
& =0 .
\end{aligned}
$$

Hence, $\widehat{x} \equiv 0$. And so, $x=0$. 
The case of a nonnormal hyponormal bilateral weighted shift is similar using the bounded point evaluations in the sense of A.L. Sheilds [7].

Remark 3.8. Using the same proof of this theorem, one can see that for every injective unilateral weighted shift $T, \operatorname{cl}\left(B_{a}(T)\right) \subset \sigma_{T}(x)$ for every nonzero element $x \in H$. The same holds for every bilateral weighted shift.

\section{ACKNOWLEDGMENTS}

This work has been partially supported by The Abdus Salam International Centre for Theoretical Physics, Trieste, Italy. The first and last authors are grateful to the mathematics section of the Abdus Salam ICTP.

\section{REFERENCES}

[1] I. Colojoara and C. Foias, Theory of Generalized Spectral Operators, Gordon and Breach, New York, 1968. MR 52:15085

[2] J.B. Conway, The Theory of Subnormal Operators, volume 36 of Mathematical Surveys and Monographs. American Mathematical Society, Providence, R.I., 1991. MR 92h:47026

[3] I. Erdelyi and R. Lange, Spectral Decomposition on Banach Spaces, Lecture Notes in Mathematics, vol. 623, Springer-Verlag, New York, (1977). MR 58:2432

[4] M. Radjabalipour, Ranges of Hyponormal Operators, Illinois J. Math. 21(1977), 70-75. MR 56:6449

[5] M. Raphael, Quasisimilarity and Essential Spectra for Subnormal Operators, Indiana Univ. Math. J. 31(1982), 243-246. MR 83d:47031

[6] W. C. Ridge, Approximate Point Spectrum of a Weighted Shift, Trans. Amer. Math. Soc. 147(1970), 349-356. MR 40:7843

[7] A. L. Shields, Weighted Shift Operators and Analytic Function Theory, in Topics in Operator Theory, Mathematical Surveys, no. 13 (ed. C. Pearcy), pp. 49-128. American Mathematical Society, Providence, Rhode Island, 1974. MR 50:14341

[8] J. G. Stampfli, A Local Spectral Theory for Operators. V: Spectral Subspaces for Hyponormal Operators, Trans. Amer. Math. Soc. 21(1976), 285-296. MR 54:8339

[9] T. T. Trent, $H^{2}(\mu)$ Spaces and Bounded Point Evaluations, Pac. J. Math. 80(1979), 279-292. MR 81j:30054

[10] L. R. Williams, Bounded Point Evaluations and Local Spectra of Cyclic Hyponormal Operators, Dynamic Systems and Applications 3(1994), 103-112. MR 95i:47008

[11] L.R. Williams, The Local Spectra of Pure Quasinormal Operators, J. Math Anal. Appl. 187(1994), 842-850. MR 95h:47029

The Abdus Salam International Centre for Theoretical Physics, Trieste, Italy

E-mail address: bourhim@ictp.trieste.it

Current address: Département de Mathématiques, Université Mohamed V, B.P. 1014, Rabat, Morocco

E-mail address: abourhim@fsr.ac.ma

The Abdus Salam International Centre for Theoretical Physics, Trieste, Italy

E-mail address: chidume@ictp.trieste.it

Département de Mathématiques, Université Mohamed V, B.P. 1014, Rabat, Morocco

E-mail address: zerouali@fsr.ac.ma 